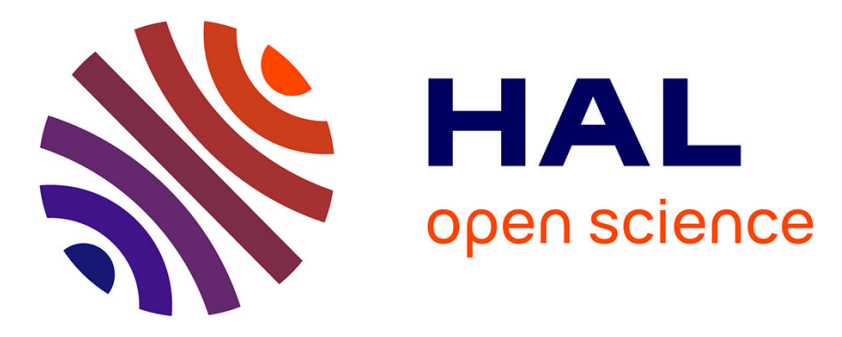

\title{
Revisiting Pressure-Induced Transitions in Mesoporous Anatase TiO2
}

Denis Machon, Laurence Bois, Défi Jr Jubgang Fandio, Quentin Martinet, Alexis Forestier, Sylvie Le Floch, Jérémie Margueritat, Vittoria Pischedda, Denis Morris, Lucien Saviot

\section{To cite this version:}

Denis Machon, Laurence Bois, Défi Jr Jubgang Fandio, Quentin Martinet, Alexis Forestier, et al.. Revisiting Pressure-Induced Transitions in Mesoporous Anatase TiO2. Journal of Physical Chemistry C, 2019, 123 (38), pp.23488-23496. 10.1021/acs.jpcc.9b05650 . hal-02283188

\section{HAL Id: hal-02283188 https://hal.science/hal-02283188}

Submitted on 20 May 2020

HAL is a multi-disciplinary open access archive for the deposit and dissemination of scientific research documents, whether they are published or not. The documents may come from teaching and research institutions in France or abroad, or from public or private research centers.
L'archive ouverte pluridisciplinaire HAL, est destinée au dépôt et à la diffusion de documents scientifiques de niveau recherche, publiés ou non, émanant des établissements d'enseignement et de recherche français ou étrangers, des laboratoires publics ou privés. 


\title{
Revisiting Pressure-induced Transitions in Mesoporous Anatase $\mathrm{TiO}_{2}$
}

\author{
Denis Machon ${ }^{1,2, *}$, Laurence Bois ${ }^{3}$, Défi Jr Jubgang Fandio ${ }^{2,4}$, Quentin Martinet ${ }^{1}$, Alexis \\ Forestier $^{1}$, Sylvie Le Floch ${ }^{1}$, Jérémie Margueritat ${ }^{1}$, Vittoria Pischedda ${ }^{1}$, Denis Morris ${ }^{2,4}$, \\ Lucien Saviot ${ }^{5}$
}

1. Univ Lyon, Université Claude Bernard Lyon 1, CNRS, Institut Lumière Matière, F-69622, Villeurbanne, France

2. Laboratoire Nanotechnologies et Nanosystèmes (LN2), CNRS UMI-3463, Université de Sherbrooke, Institut Interdisciplinaire d'Innovation Technologique (3IT), Sherbrooke QC J1K 0A5, Québec, Canada

3. Laboratoire Multimatériaux et Interfaces, UMR CNRS 5615, Université Claude Bernard Lyon 1, Lyon 69007, France

4. Département de Physique et Regroupement Québécois sur les Matériaux de Pointe, Université de Sherbrooke, Québec, J1K2R1, Canada

5. Laboratoire Interdisciplinaire Carnot de Bourgogne, UMR 6303 CNRS Université de Bourgogne Franche-Comté, 9 Av. A. Savary, BP 47870, F-21078 Dijon Cedex ,France

* Corresponding author : denis.machon@univ-lyon1.fr

\begin{abstract}
Mesoporous materials may be considered as the negative image of a collection of nanoparticles. In both cases, the large surface area induces an increase of the system energy. Pressure-induced phase transitions are used to study the differences between mesoporous $\mathrm{TiO}_{2}$ and a collection of $\mathrm{TiO}_{2}$ nanoparticles. The synthesis and characterization of mesoporous $\mathrm{TiO}_{2}$ with a nanosponge-like structure are presented and the inputs of different spectroscopic techniques (Terahertz, Brillouin and Raman) are highlighted. The results show that the phase stability of mesoporous $\mathrm{TiO}_{2}$ under pressure is very similar to that observed in nanoparticles.
\end{abstract}




\section{Introduction}

Mesoporous materials may be an alternative to nanoparticle-based materials for manufacturing (obtaining ceramics from powders of nanoparticles keeping the interesting related properties) and health implications (nanotoxicology). The high surface-to-volume ratio in both cases enhances the properties related to surface effects such as catalysis, Li insertion, reactivity, batteries and supercapacitors ${ }^{1,2}$. In addition, as in nanoparticles, interfaces and point defects at the surface of mesoporous materials induce additional contributions to the total energy of the system, leading to a new energy landscape that modifies phase stabilities and promotes emergence of new phases with potentially interesting properties.

Following the same interest of using high pressure on nanoparticles ${ }^{3,4}$, the thermodynamics of mesoporous materials can be explored by means of pressure-induced transformations. Understanding phase stabilities in materials with high surface-to-volume ratio is a key to control the properties associated with the new engineered structures. Thus, the exploration of the energy landscapes during compression can lead to polymorphic transition, transformation to metastable state and polyamorphism ${ }^{5}$. For instance, mesoporous silicon and germanium undergo pressure-induced amorphization on compression and a poly-amorphic transformation during subsequent decompression ${ }^{6,7}$.

$\mathrm{TiO}_{2}$ is a major oxide material because its properties are essential for many applications ${ }^{8}$ but it is also a typical example of material in which interfaces dictate the phase stability ${ }^{9}$. The interplay between the morphology (nanoparticles, nanowire, nanoribbons) and the

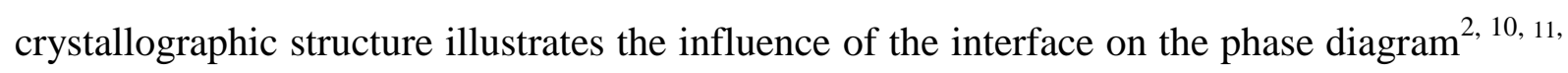
12 .

The stable structure in bulk $\mathrm{TiO}_{2}$ is the rutile-type one. Under increasing pressure, the thermodynamic sequence corresponds to the following set of structures: rutile $-\alpha-\mathrm{PbO}_{2}-$ baddeleyite. The first phase transition was reported at $2.6 \mathrm{GPa}^{13}, 7 \mathrm{GPa}^{14}, 10 \mathrm{GPa}^{15}$ and the second one at $15 \mathrm{GPa}^{15}$. However, often the intermediate $\alpha-\mathrm{PbO}_{2}$ phase is bypassed and a direct rutile-to-baddeleyite transformation can be observed at $\sim 12 \mathrm{GPa}^{16,17}$. The variability observed on the pressure-induced transformations is connected to kinetics that could be related to crystallographic relationships between the structures ${ }^{12}$. During decompression, the baddeleyite structure transforms into $\alpha-\mathrm{PbO}_{2}$ phase at around $7 \mathrm{GPa}$. This phase is preserved in a metastable state down to ambient pressure ${ }^{17}$. 
At the nanoscale, there is a crossing of phase stability because the surface energy favors the anatase structure usually observed in nanoparticles ${ }^{18}$. High-pressure experiments on such samples have shown that the anatase structure is preserved up to $18 \mathrm{GPa}$ before transforming to the high-pressure baddeleyite structure ${ }^{9,19}$. An interesting phenomenon has been evidenced for nanoparticles smaller than $10 \mathrm{~nm}$. Under pressure, the initial crystalline structure undergoes transformation to an amorphous state, a transition that is absent in the case of bulk $\mathrm{TiO}_{2}$. This size-dependent pressure-induced amorphization has been extensively investigated $^{20,21}$ together with poly-amorphic transformations ${ }^{22}$. The amorphization has been shown to result from the density of defects at the surface which increases the interface energy $^{9,23}$.

The synthesis of mesoporous materials is a means of creating interfaces and defects, and the mesoporous materials may be considered as the negative image of a collection of nanoparticles ${ }^{7}$. A question that arises is whether the surface energy component of mesoporous materials is equivalent to that of a collection of nanoparticles and leads to similar metastable states. If the surface area can be considered equivalent, the curvature of the interface at the nanoscale is different and may lead to other effects ${ }^{7}$.

Mesoporous rutile $\mathrm{TiO}_{2}$ has been studied under high pressure ${ }^{24}$. A transformation to the baddeleyite phase started at $10.8 \mathrm{GPa}$ and was completed at $26.1 \mathrm{GPa}$. The rather low transition pressure has been attributed to microscopic strains that develop into the porous structure. This point has been discussed in ref ${ }^{7}$. In the case of nanoparticles, the stress can be distributed continuously and smoothly over the spherical shape. On the contrary, discontinuities in the mesoporous network with hyperbolic geometry will lead to inhomogeneities in the stress distribution. During decompression, the baddeleyite phase transformed into the $\alpha-\mathrm{PbO}_{2}$ structure below $\sim 6 \mathrm{GPa}^{24}$. Surprisingly, the mesoporous structure remained intact after a complete pressure cycle. According to the authors, this could be due to the invasion by the liquid pressure-transmitting medium (mixture of ethanol and methanol).

Mesoporous $\mathrm{TiO}_{2}$ with anatase-type structure was been studied by the same group by Raman spectroscopy ${ }^{25}$. They reported a phase transition from anatase to baddeleyite in the range 15$18 \mathrm{GPa}$ followed by a reversible pressure-induced amorphization (PIA) above $20.5 \mathrm{GPa}$. On decompression, the $\alpha-\mathrm{PbO}_{2}$ phase is obtained. Despite this interesting result, the role of porosity is not yet clearly defined because the nature of the porous material was ill-defined. Their sample consisted of an agglomeration of $30 \mathrm{~nm}$-diameter particles "constructing a nanoporous structure", according to the authors ${ }^{25}$. However, neither pore size distribution nor 
the porosity were clearly mentioned. This information is mandatory, as the impact of the morphology is still not clear. For instance, in 1D nanostructures, it appears that the initial phase at ambient pressure is the main factor influencing the pressure-induced transitions sequence rather than morphology ${ }^{12}$.

In the present work, we want to clarify the possible role played by the mesostructure on the pressure-induced polymorphism. Therefore, the first part of this article is devoted to characterizing the texture of the mesoporous material with highlights on the key information provided by a given set of spectroscopic techniques (Terahertz, Brillouin and Raman). The second part deals with the pressure-induced evolution monitored using Brillouin and Raman spectroscopies. A comparison with the results obtained on 7-nm nanoparticles is done and it is shown that the interface effects are very similar in both cases.

\section{Experimental}

Mesoporous titania samples were prepared by a sol-gel process with the evaporation induced self-assembly method proposed by Brinker et al. $^{26}$. The poly(ethylene oxide)poly(propylene oxide)-poly(ethylene oxide) triblock copolymer $\left(\mathrm{P}-123,(\mathrm{EO})_{20^{-}}(\mathrm{PO})_{70^{-}}\right.$ $\left.\left.(\mathrm{EO})_{20}\right)\right)$ was used as the template and $\mathrm{Ti}\left(\mathrm{OBu}^{\mathrm{n}}\right)_{4}$ as the inorganic precursor ${ }^{27,28}$.

A solution of P-123 $(1 \mathrm{~g})$ in ethanol $(12 \mathrm{~g})$ is added to a solution of $\operatorname{Ti}\left(\mathrm{OBu}^{\mathrm{n}}\right)_{4}(3.4 \mathrm{~g})$ in concentrated $\mathrm{HCl}(3.2 \mathrm{~g})$. The solution is heated at $50{ }^{\circ} \mathrm{C}$ for 2 days, and the obtained solid is calcinated at $350^{\circ} \mathrm{C}$ for $2 \mathrm{~h}$, with a heating ramp of $1{ }^{\circ} \mathrm{C} \min ^{-1}$. The detailed synthesis has been reported elsewhere ${ }^{29,30}$.

Characterization of the texture was realized using nitrogen adsorption/desorption isotherms on a BelsorpMini (Bel Instruments, Japan). Prior analysis, samples were outgassed under vacuum $\left(100^{\circ} \mathrm{C}\right.$ for $\left.4 \mathrm{~h}\right)$. The pore size distribution (max radius) and the mesoporous volume were calculated from the adsorption branch of the isotherms using the Barrett-JoynerHalenda $(\mathrm{BJH})$ method. The total porous volume was measured at $\mathrm{P} / \mathrm{P}_{0}=0.98$.

TEM pictures were acquired on a Topcon instrument operating at $200 \mathrm{kV}$ in the "Institut Lumière Matière". Prior to the observations, the sample was ground and deposited directly on a copper grid coated with a holey carbon film.

Raman spectra were obtained using a LabRAM HR Evolution Raman Spectrometer (Horiba Scientific) using an excitation wavelength of $532 \mathrm{~nm}$. This spectrometer is equipped with a commercial ultralow frequency module allowing to reach frequency as low as $7 \mathrm{~cm}^{-1}$. The laser excitation power was limited to $5 \mathrm{~mW}$ at the entrance of the diamond anvil cell 
(DAC) to avoid heating. The beam was focused on the sample using a long working distance (21 mm) 50x objective, with beam diameter $\sim 2 \mu \mathrm{m}$ at the surface of the sample. The scattered light was collected using the same objective (back-scattering geometry).

Micro-Brillouin experiments were carried out with a homemade setup that combines a tandem Fabry-Perot interferometer equipped with a photomultiplier tube with an inverted confocal microscope. The Brillouin spectrometer is coupled to a solid-state laser source emitting at $532 \mathrm{~nm}$. The laser power was limited at $10 \mathrm{~mW}$ to avoid damaging the sample. Measurements were done in the backscattering geometry using a 50X long working distance microscope objective.

The THz-TDS setup was coupled to a Ti:sapphire oscillator which delivers 70 fs laser pulses centered at $790 \mathrm{~nm}$ at a $82-\mathrm{MHz}$ repetition rate. The laser beam was split into two distinct parts, an excitation beam used to generate the broadband $\mathrm{THz}$ radiation and a probing beam used to detect $\mathrm{THz}$ pulses transmitted through the sample. A home-made GaAs photoconductive antenna has been used as the THz source. This antenna has been illuminated with a laser beam power of about $50 \mathrm{~mW}$. Hemispherical silicon lenses, with a 4-mm diameter, were used to collect and refocus the $\mathrm{THz}$ radiation. The THz-probe beam was confined in a moisture-free environment (pressure <1 mbar) and a four off-axis parabolic mirrors configuration was used. The $\mathrm{THz}$ radiation was focused on the sample surface on a spot diameter of about $2 \mathrm{~mm}$. The transmitted THz pulses were detected using electro-optic sampling in a 0.5 -mm-thick ZnTe crystal. The THz-probe beam was chopped at $1350 \mathrm{~Hz}$ and the $\mathrm{THz}$ signal was measured by means of a lock-in amplifier. All measurements were made at room temperature ${ }^{31}$.

High pressure was generated using a membrane DAC with low-fluorescence diamonds. Mesoporous $\mathrm{TiO}_{2}$ samples were placed into a $125 \mu \mathrm{m}$ chamber drilled in an indented stainless-steel gasket. Paraffin oil was used as the pressure-transmitting medium (PTM). With this PTM hydrostaticity is ensured up to $4-5 \mathrm{GPa}^{32}$. The pressure was probed by the shift of the R1 fluorescence line of a small ruby chip.

\section{Results and discussion}

\section{Sample characterization at ambient conditions}

$\mathrm{X}$-ray diffraction pattern is shown in figure 1 . It can be indexed using the anatase-type structure (space group No $141-I_{1} /$ amd) with a $\sim 3.78 \AA$ and $\mathrm{c} \sim 9.51 \AA$. No preferred orientation can be deduced from the intensity analysis. 


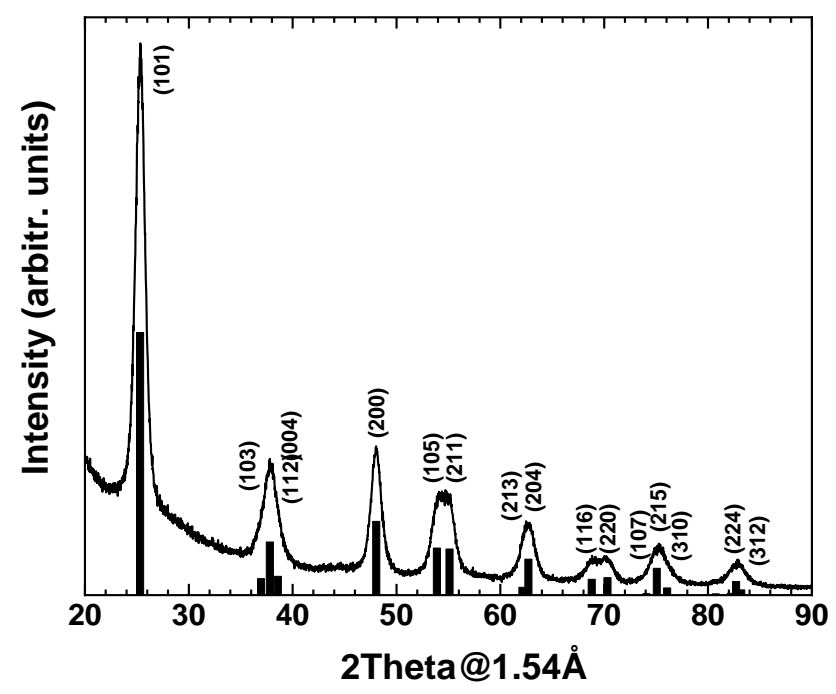

Figure 1 - X-ray diffraction of mesoporous $\mathrm{TiO}_{2}$. The data is indexed using the anatase-type structure with $\mathrm{a}=3.78 \AA$ and $\mathrm{c}=9.51 \AA$.

Transmission electron microscopy images are shown in figure 2. The images show the morphology of the mesostructured anatase $\mathrm{TiO}_{2}$ material with a distinct mesoporous texture that can be described as nanosponge-like. The structure is not an agglomeration of nanoparticles. TEM image at high magnification shows obvious 4-6 nm pores.

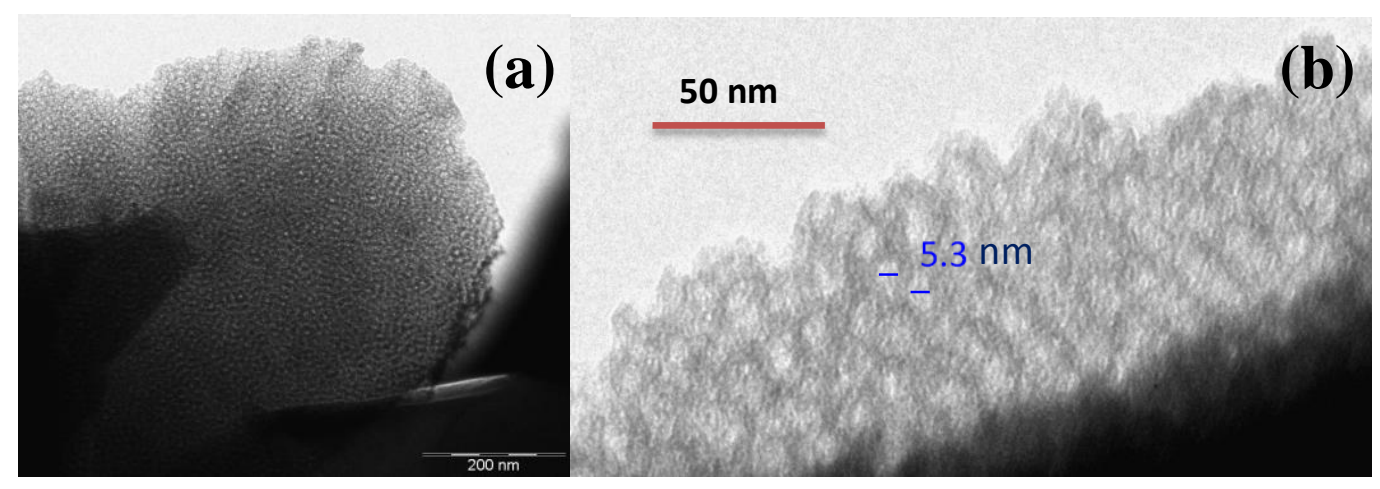

Figure 2 - Transmission Electron Microscopy images of mesoporous titania films with (a) low and (b) high magnification 


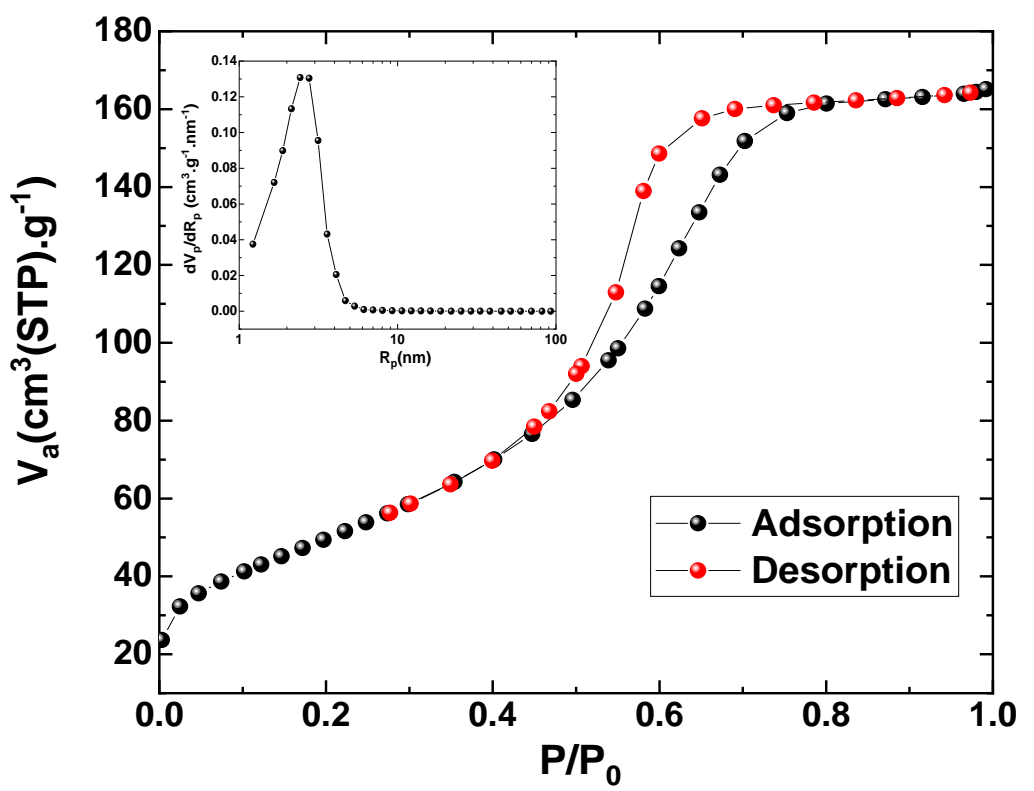

Figure $3-\mathrm{N}_{2}$ adsorption-desorption isotherms and BJH analysis from the adsorption branch (inset)

The $\mathrm{N}_{2}$ adsorption/desorption isotherms and $\mathrm{BJH}$ plots are shown in figure 3. The physisorption isotherms correspond to type IV and the hysteresis to type $\mathrm{H} 2$ according to the IUPAC classification ${ }^{33}$. This behavior is characteristic of a mesoporous material. The pore size distribution $(\mathrm{BJH})$ is centered at $5 \mathrm{~nm}$ in agreement with the TEM images (Figure 2). The mesoporous volume is estimated at $0.26 \mathrm{~cm}^{3} / \mathrm{g}$. By using the density of anatase $(\mathrm{d}=4.23$ $\mathrm{g} / \mathrm{cm}^{3}$ ), this gives a porosity $\varepsilon \sim 52 \%$. This corresponds to the open porosity, which is a lower limit of the total porosity of the sample.

Total porosity is estimated using two distinct spectroscopic methods. Brillouin spectroscopy probes the sound velocity in a material through its light scattering spectrum, and terahertz spectroscopy probes dielectric properties of the material using a transmission configuration. 
Figure 4 compares the waveform of the $\mathrm{THz}$ pulses propagating in the vacuum (reference trace, black line) to that of the pulses transmitted through a $2 \mathrm{~mm}$-thick porous $\mathrm{TiO}_{2}$ sample. The knowledge of the sample thickness and the delay $\Delta t=11.528 \mathrm{ps}$ between the maxima of these $\mathrm{THz}$ traces makes it possible to extract the group refractive index of the mesoporous film. The estimated value, $n=2.73$, lies somewhere between the refractive index of air $\left(\mathrm{n}_{\text {air }}=1\right)$ and the one of bulk $\mathrm{TiO}_{2}\left(N_{=} 5.84\right)$ at $\mathrm{THz}$ frequencies ${ }^{34}$. These spectroscopic data can be analyzed using an effective medium approximation. The Maxwell-Garnett and the Bruggeman models ${ }^{35}$ are commonly used to treat such systems. In our case, as the estimated porous fraction being comparable to that of the host material, the Bruggeman approximation is well adapted. The effective refractive index $n$ is related to the porosity $\varepsilon$ through the following equation ${ }^{36}$ :

$$
1-\varepsilon=\frac{\left(1-n^{2}\right)\left(N^{2}+2 n^{2}\right)}{3 n^{2}\left(1-N^{2}\right)}
$$

where $N$ is the refractive index of the bulk material. Using this equation, the porosity is estimated at $\varepsilon \sim 57 \%$.

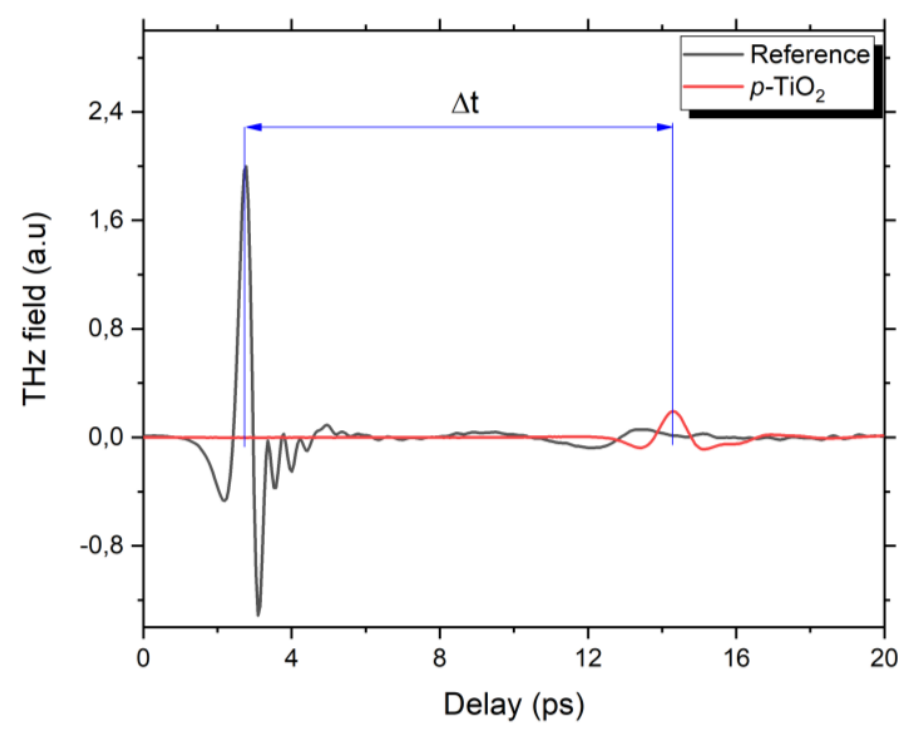

Figure 4 - THz pulse for both reference and 2-mm-thick porous $\mathrm{TiO}_{2}$ sample. 


\section{Brillouin spectroscopy measurements}

The Brillouin spectrum of the mesoporous film is presented in figure 5. The observed peak is centered at $v_{B}=18 \mathrm{GHz}$. The Brillouin frequency is given by

$$
v_{B}=\frac{2 n(\varepsilon) V(\varepsilon)}{\lambda}
$$

Where $n(\varepsilon)$ is the effective refractive index and $V(\varepsilon)$ is the sound velocity in the material. The function $V(\varepsilon)$ is unknown for $\mathrm{TiO}_{2}$ but in the case of porous silicon, it has been shown that $V(\varepsilon)=V_{0}(1-\varepsilon)^{m}$ with $m=1.083$ where $V_{0}$ is the sound velocity in the bulk material $\left(V_{0}\right.$ $=8367 \mathrm{~m} . \mathrm{s}^{-1}$ for anatase $\left.\mathrm{TiO}_{2}\right)^{36}$. Using this relation and the Bruggeman equation $(N=2.6$ at $\lambda$ $=532 \mathrm{~nm}$ ) giving $n(\varepsilon)$ as in the case of the terahertz spectroscopy, one finds $n=1.58$ and a porosity $\varepsilon \sim 60 \%$ in very good agreement with the $\mathrm{THz}$ spectroscopy results.

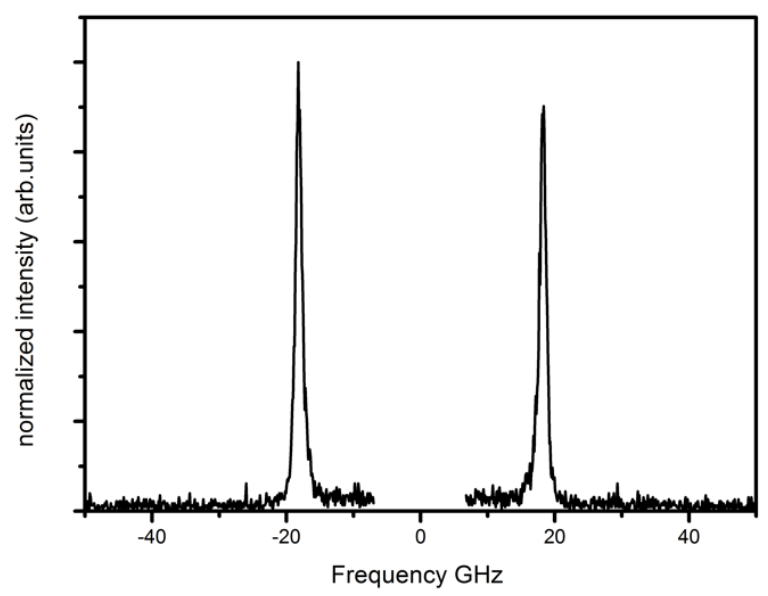

Figure 5 - Brillouin spectrum of mesoporous $\mathrm{TiO}_{2}$.

\section{Raman Spectroscopy measurements}

Raman spectrum of as-made mesoporous $\mathrm{TiO}_{2}$ sample is shown in figure 6. Five Raman peaks are measured in the range $60-800 \mathrm{~cm}^{-1}$ and are assigned to the anatase structure ${ }^{37}$. In bulk $\mathrm{TiO}_{2}$ anatase, the vibrational representation of the normal modes at the center of the Brillouin zone is given by

$$
\Gamma_{v i b}=A_{1 g} \oplus A_{2 u} \oplus 2 B_{1 g} \oplus B_{2 u} \oplus 3 E_{g} \oplus 2 E_{u}
$$

Only the modes $\mathrm{A}_{1 \mathrm{~g}}, \mathrm{~B}_{1 \mathrm{~g}}$ and $\mathrm{E}_{\mathrm{g}}$ are Raman active. The peak positions were measured at 144 $\mathrm{cm}^{-1}\left(\mathrm{E}_{\mathrm{g}}\right), 197 \mathrm{~cm}^{-1}\left(\mathrm{E}_{\mathrm{g}}\right), 400 \mathrm{~cm}^{-1}\left(\mathrm{~B}_{1 \mathrm{~g}}\right), 507 \mathrm{~cm}^{-1}\left(\mathrm{~A}_{1 \mathrm{~g}}\right), 519 \mathrm{~cm}^{-1}\left(\mathrm{~B}_{1 \mathrm{~g}}\right)$ and $640 \mathrm{~cm}^{-1}\left(\mathrm{E}_{\mathrm{g}}\right)$. 
It is interesting to note that the $\mathrm{E}_{\mathrm{g}}$ mode at $197 \mathrm{~cm}^{-1}$ is clearly visible. This indicates that there is no optical phonon confinement effect for the optical phonons, contrary to what is observed in the case of nanoparticles (see Supp info ${ }^{31}$ ). However, additional low-frequency features are observed similarly to what is observed in $\mathrm{TiO}_{2}$ nanoparticles ${ }^{38}$. These peaks are associated with the anatase structure as they are sensitive to phase transitions as shown in the following section dedicated to high-pressure transformations. In the case of $\mathrm{TiO}_{2}$ nanoparticles, a single low-frequency peak attributed to confined acoustic phonons is generally observed and is assigned to the fundamental quadrupolar mode (spheroidal vibration with angular momentum $l=2$ noted $\left.S_{2}^{1}\right)^{38}$.

In our spectrum, two low-frequency peaks are observed at $\sim 12.6 \mathrm{~cm}^{-1}$ and $\sim 22.7 \mathrm{~cm}^{-1}$. In a simple approach, these peaks would correspond to anatase nanospheres with radius 4.8 and $8.7 \mathrm{~nm}$, respectively. However, our mesoporous samples are not made of stacked nanoparticles but correspond to a nanosponge-like structure according to the TEM images (Figure 2). Therefore, we compared these frequencies with those of quadrupolar-like vibrations calculated $^{39}$ for other nanostructure shapes that can match the TEM images. The diameters for isotropic anatase $\mathrm{TiO}_{2}$ spheres, shells, cylinders and tubes having a quadrupolarlike vibration at 12.6 and $22.7 \mathrm{~cm}^{-1}$ are given in Table $\mathrm{S}^{31}$. For simplicity, the inner diameter for the empty spheres and tubes was fixed at $5.3 \mathrm{~nm}$ according to the TEM images (Figure 2). In this approach, we neglect the mechanical coupling between adjacent sub-structures of the porous material. Using this simple picture, the $22.7 \mathrm{~cm}^{-1}$ low-frequency peak can be assigned to vibrations of any of these structures. The calculated diameters match at least approximately the sizes observed in the TEM images. On the contrary, the diameters required to reproduce the $12.6 \mathrm{~cm}^{-1}$ peak are about twice as large and do not match with features observed in the TEM images. Therefore, this low-frequency peak is difficult to assign in this simple approach. Raman features at frequencies lower than those of quadrupolar-like vibrations appear when a mechanical coupling between neighbor nanostructures exists. This has already been observed for coupled nanospheres ${ }^{40}$. Therefore, the $12.6 \mathrm{~cm}^{-1}$ peak may be assigned to such coupled vibrations. However, a precise description of the porous nanostructure is required to reproduce this frequency numerically. This goes beyond the scope of this work. Still, the previous assignment will be used in the following: modifications (or the lack thereof) of the low-frequency spectra will be related to changes (or the lack thereof) of the mesoporous structure. 


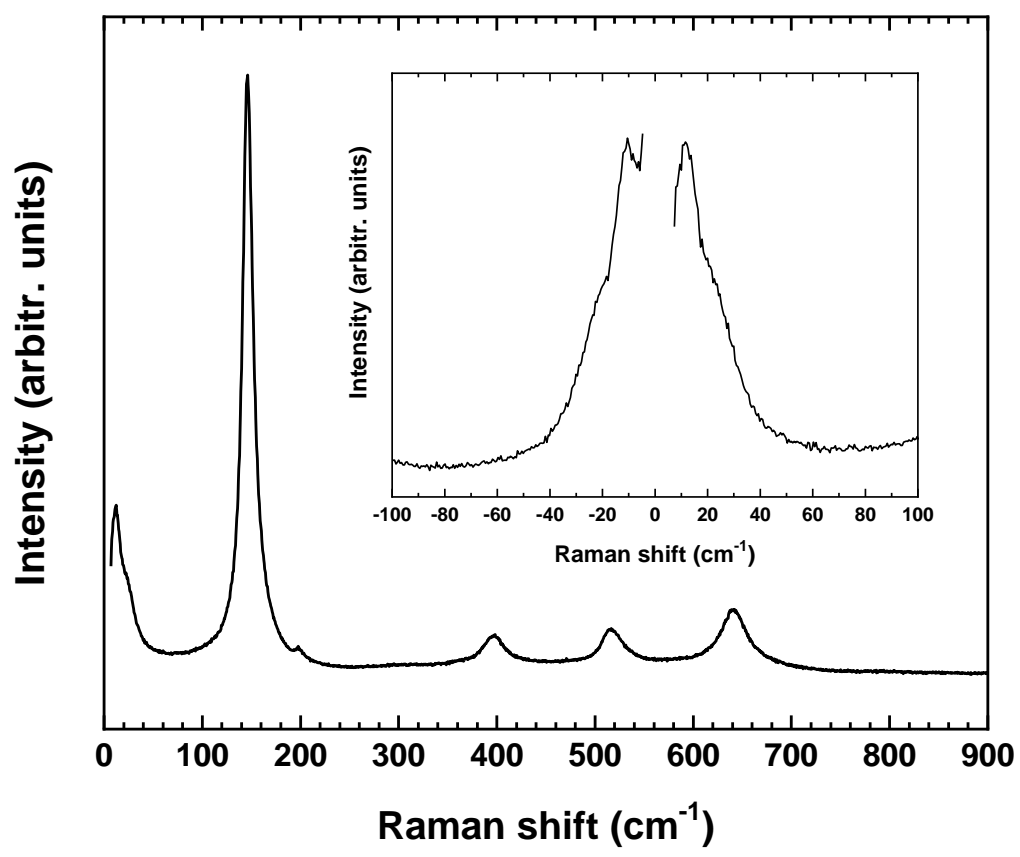

Figure 6 - Raman spectrum of porous $\mathrm{TiO}_{2}$. The inset shows the low-frequency range where confined acoustic phonons give rise to additional features.

\section{High-pressure experiments on mesoporous $\mathrm{TiO}_{2}$}

\section{Brillouin spectroscopy measurements}

Brillouin spectroscopy is compatible with the high-pressure environment and it is possible to follow the Brillouin peak of mesoporous $\mathrm{TiO}_{2}$ in situ as a function of pressure (Figure 7a). Peak position was monitored up to $12 \mathrm{GPa}$. In this pressure range, the structure remains the anatase-type one according to Raman spectroscopy experiments reported in the next section. The Brillouin peak position is plotted as a function of the pressure in figure $7 \mathrm{~b}$, along with the corresponding porosity obtained from equation 2 . The value of the porosity decreases rapidly with the increasing pressure and reaches $20-25 \%$ at high-pressure. During decompression, the porosity shows some reversibility. This is in agreement with the results obtained on pressurecycled mesoporous rutile $\mathrm{TiO}_{2}{ }^{24}$ for which TEM analysis before and after the pressure cycle showed that the porosity is preserved even after a treatment at $38.8 \mathrm{GPa}$. This effect has been attributed to the penetration of the pressure-transmitting medium (PTM) into the mesopores. The PTM used in ref ${ }^{24}$ was an alcohol mixture and in our case, it was a paraffin oil, a more viscous liquid. However, a parameter that determined the penetration of the liquid is the wettability and titania has shown little resistance to complete wetting of paraffin oil ${ }^{41}$. In 
addition, moderate pressures (compared to those applied in the present study) increase wettability ${ }^{41}$. In conclusion, as in ref ${ }^{24}$, it can be assumed that the preservation of the porosity during pressure cycling may be due to the invasion of the mesopores by the liquid pressure transmitting medium.

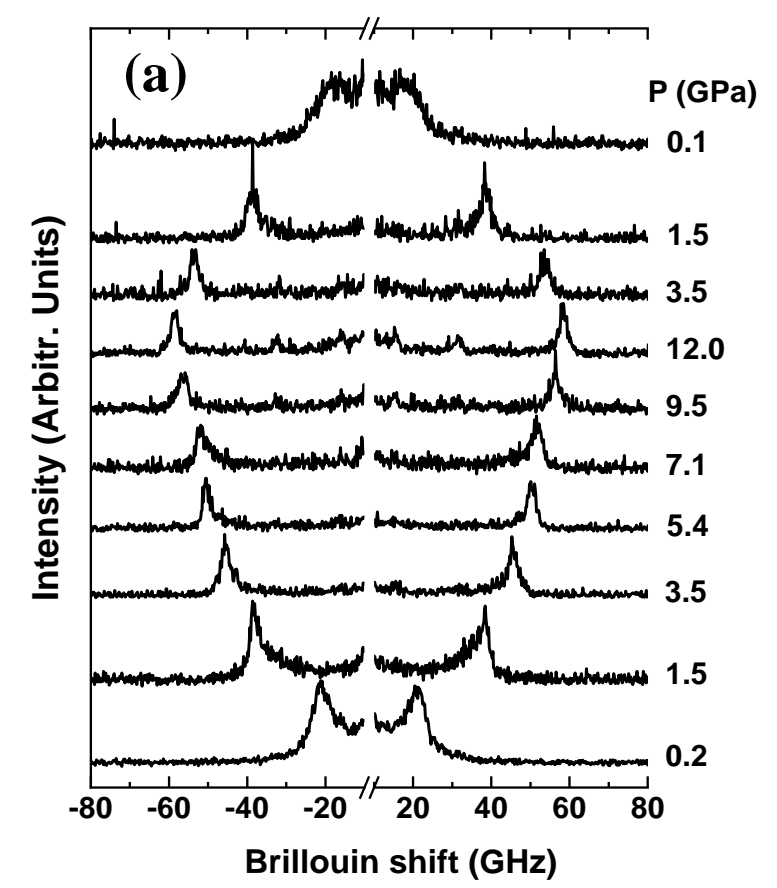

(b)

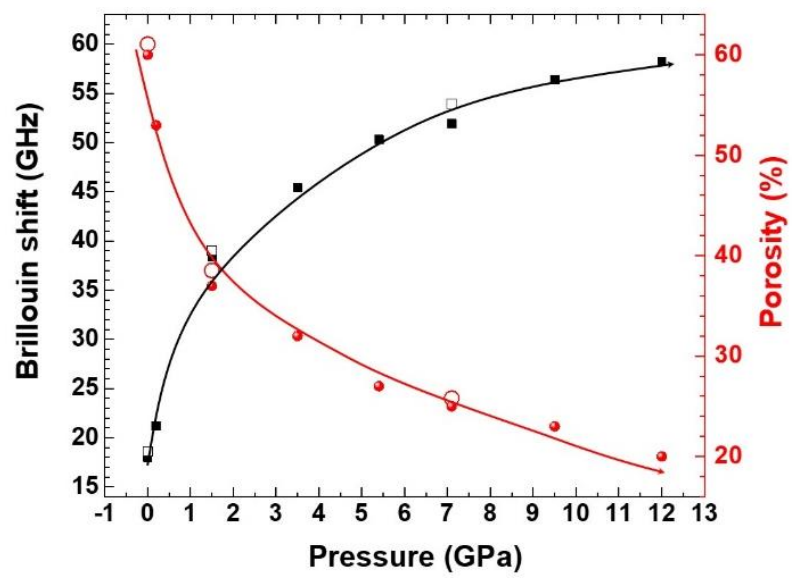

Figure 7 - (a) Brillouin spectra as a function of pressure during compression (up to 12 $\mathrm{GPa} /$ decompression cycle; (b) peak position along with the corresponding porosity as a function of pressure on compression (full symbols) and decompression (open symbols).

\section{Raman spectroscopy measurements}

The evolution of the Raman spectra of mesoporous $\mathrm{TiO}_{2}$ as the pressure increases up to 28.3 GPa are shown in figure 8. Up to $13 \mathrm{GPa}$, no change is observed except for the usual blue shift of the Raman peaks. Pressure-induced evolutions of the Raman frequencies are reported in Figure $\mathrm{S} 3^{31}$ along with those obtained for nanoparticles (7 $\mathrm{nm}$ diameter) and $a b$ initio simulations $^{42}$. At $\sim 17-18 \mathrm{GPa}$, new peaks appear and develop with increasing pressure. Phase transformation is only complete above $\sim 25 \mathrm{GPa}$. The Raman bands of the high-pressure phase are weak and broad, indicating the appearance of a significant degree of disorder. However, contrary to previous report on mesoporous anatase $\mathrm{TiO}_{2}{ }^{25}$, a total amorphization of the film is not observed in our experiments, above $20.5 \mathrm{GPa}$. Comparison of the spectrum obtained at the highest pressure in this run to that obtained on $\mathrm{TiO}_{2}$ nanoparticles ( $7 \mathrm{~nm}$ diameter) at similar 
pressure is shown in figure 9. The high-pressure phase in nanoparticles has been identified as the baddeleyite structure by synchrotron X-ray diffraction ${ }^{9}$. In the case of the mesoporous sample, the spectrum is similar but shows a higher degree of disorder, maybe a coexistence of amorphous and crystalline regions. This apparent disordering may be due to internal stresses and inhomogeneous stress field because of the mesoporous morphology, as discussed in ref ${ }^{7}$. The impact of a non-homogeneous stress field on the phase transition and the genesis of complex nanostructure results from a competition between polymorphic transition and amorphization. The balance between the amorphous and crystalline domains depends on the surface and the associated defects ${ }^{23}$.

The low-frequency Raman spectra of mesoporous $\mathrm{TiO}_{2}$ under pressure are shown in figure 8 . The low-frequency peak shows very little change (linear variation of $\sim 0.06 \mathrm{~cm}^{-1}$. $\mathrm{GPa}^{-1}$ ) up to $\sim 25 \mathrm{GPa}$, which corresponds to the end of the transformation from the anatase to the disordered baddeleyite phase. This low pressure-dependency of the peak position was also observed for confined acoustic waves in $\mathrm{TiO}_{2}$ nanoparticles in agreement with ab initio calculations $^{42}$. The peak initially located at $22.7 \mathrm{~cm}^{-1}$ has a larger variation under pressure $\left(\sim 0.5 \mathrm{~cm}^{-1} . \mathrm{GPa}^{-1}\right)$ up to $10 \mathrm{GPa}$. Above this pressure, the pressure-induced change has a plateau (Figure 10). This behavior seems to be correlated with the behavior of the Brillouin peak under pressure (figure 7), an observation that supports the possibility that this lowfrequency Raman peak is related to the porous nature of the sample. The disappearance of these two peaks across the transition indicates that they are related to the initial anatase structure. 


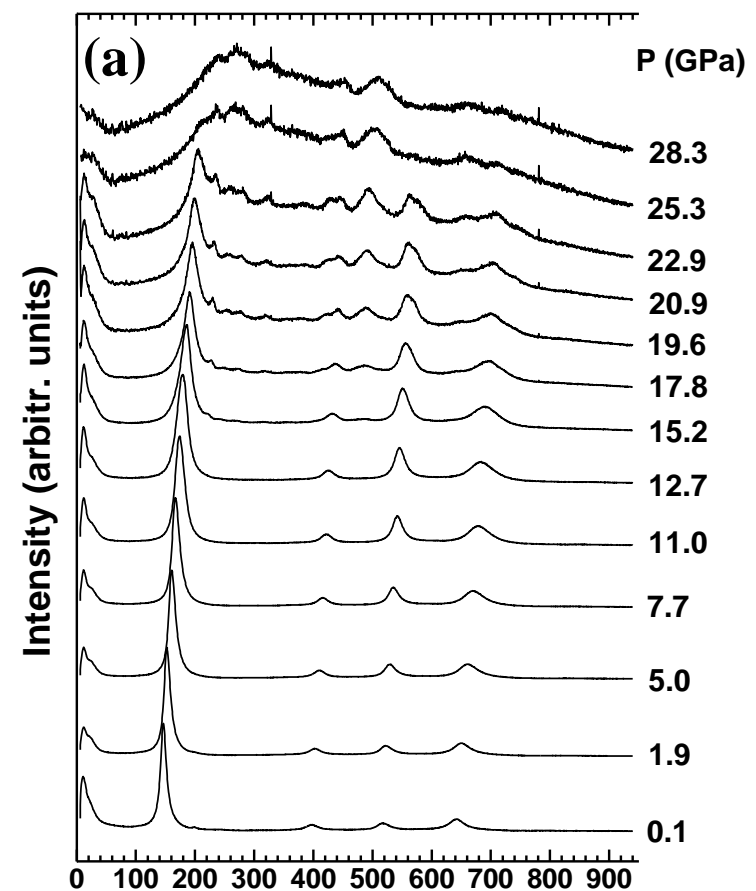

Raman shift $\left(\mathrm{cm}^{-1}\right)$

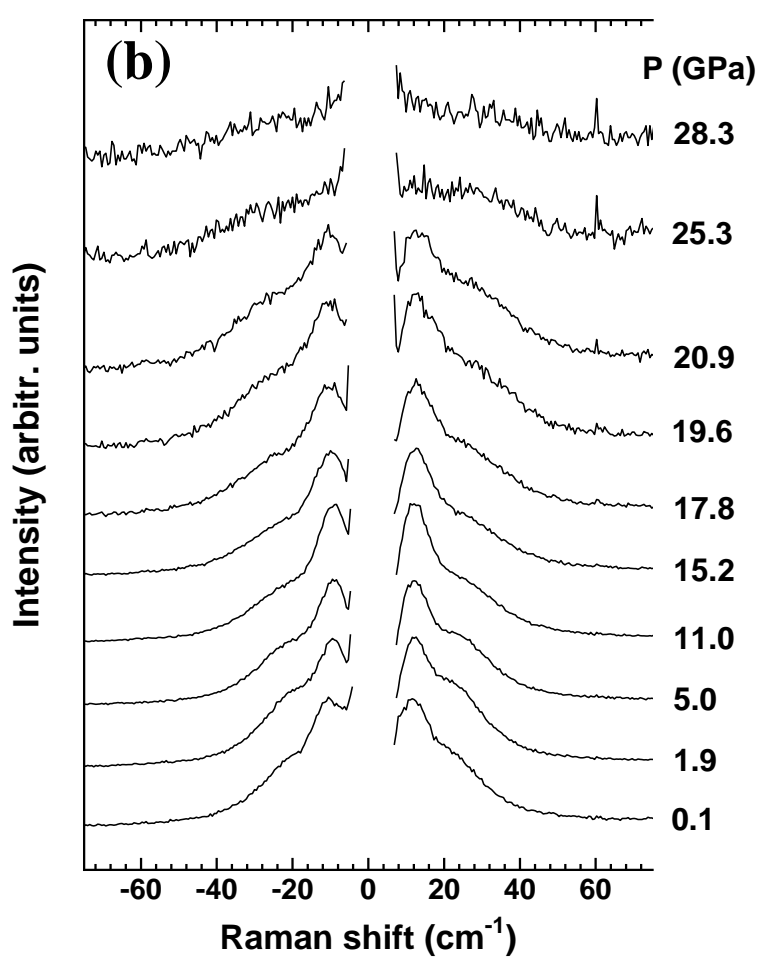

Figure 8 - Selection of Raman spectra of mesoporous $\mathrm{TiO}_{2}$ with increasing pressure for (a) the extended $0-950 \mathrm{~cm}^{-1}$ range (b) the low-frequency range $-70 ;+70 \mathrm{~cm}^{-1}$

Raman spectra were recorded during decompression (Figure 11). The disordered baddeleyite structure is observed down to $\sim 15 \mathrm{GPa}$. New features are observed at $\mathrm{P}=7.6 \mathrm{GPa}$. It is worth noting that the spectrum at $7.6 \mathrm{GPa}$ differs from the one at $3.7 \mathrm{GPa}$, indicating the appearance of an intermediate structure. Such an observation is similar to the report of an intermediate phase during decompression of anatase nanoparticles or rutile nanorods after a pressure cycle ${ }^{9,12}$. Comparison of spectra of nanoparticles and mesoporous $\mathrm{TiO}_{2}$ after a pressure cycle is shown in figure 9. The similarity of the Raman spectra indicates that the low-pressure phase corresponds to the $\alpha-\mathrm{PbO}_{2}$ structure. Interestingly, the peaks of the mesoporous sample are narrower than those of the nanoparticles despite an apparent higher degree of disorder of the high-pressure phase. This reinforces our interpretation that the internal stress distribution is highly inhomogeneous because of the hyperbolic geometry of a mesoporous structure ${ }^{7}$. On decompression, these stresses are relaxed and there is no broadening effect as it is the case for nanoparticles. 


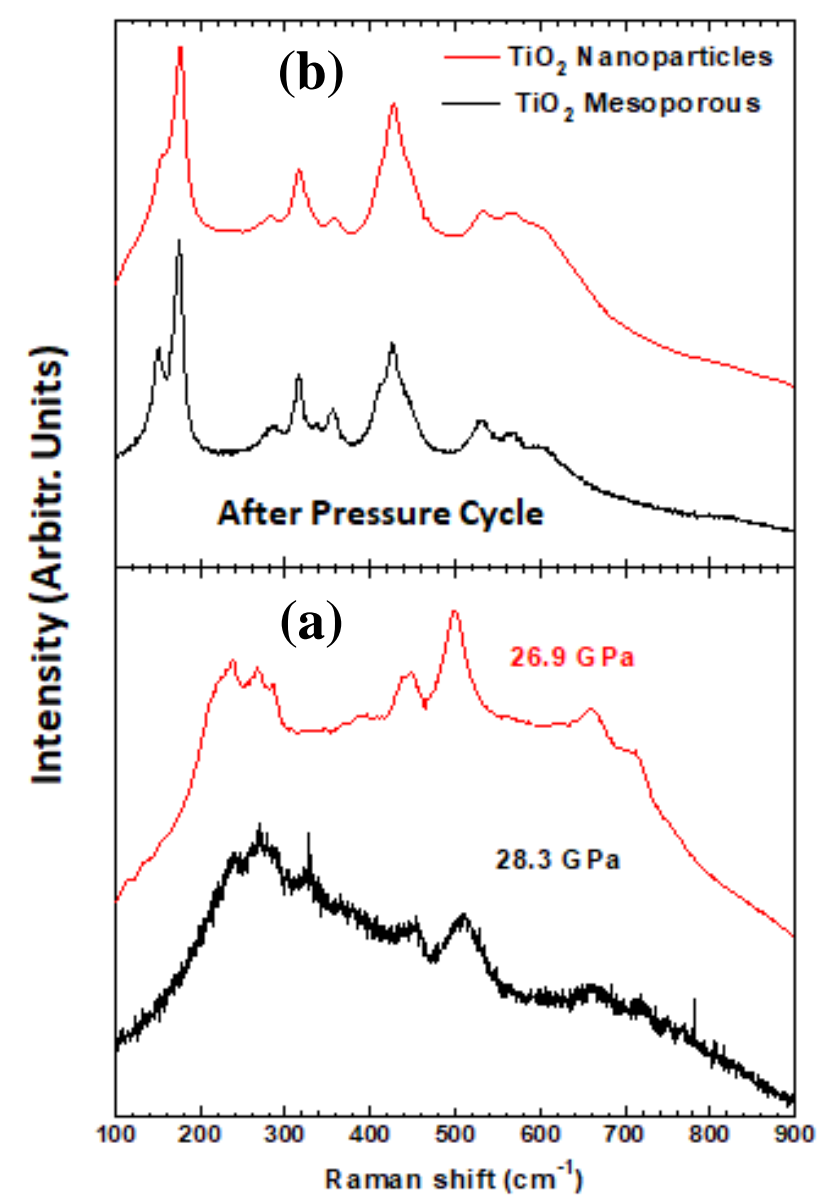

Figure 9 - Comparison of Raman spectra of a powder of $\mathrm{TiO}_{2}$ nanoparticles (7-nm diameter) (from $\operatorname{Ref}^{9}$ ) and mesoporous $\mathrm{TiO}_{2}$ at (a) high pressure (b) after pressure cycle.

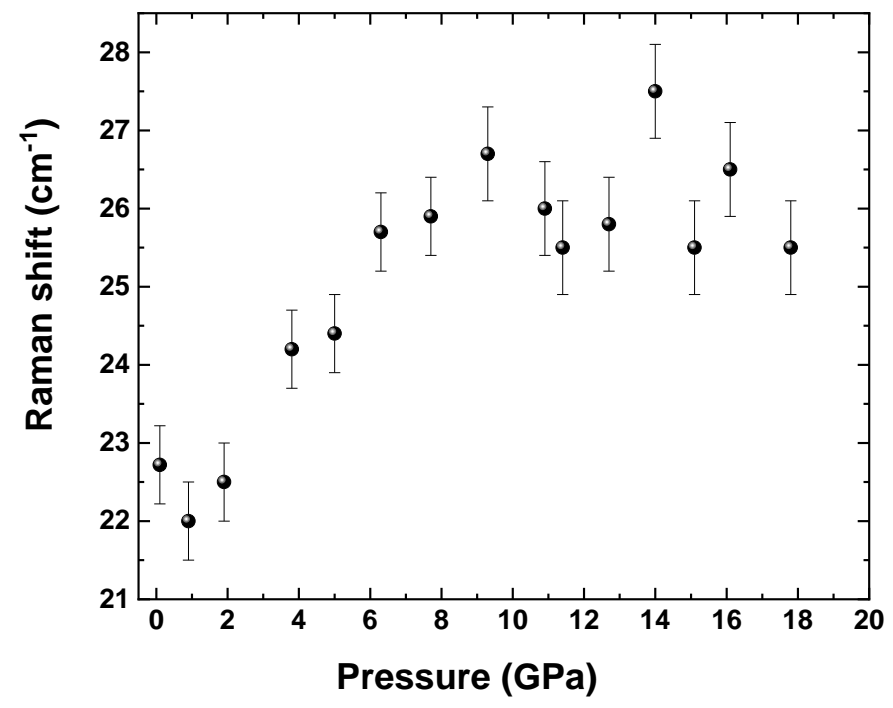

Figure 10 - Pressure-induced evolution of a low-frequency Raman mode. 
The low-frequency Raman spectra during decompression show a re-emergence of the signal below $20.8 \mathrm{GPa}$. The signal gains intensity and shifts towards low frequencies. This signature indicates that the porosity is partially preserved during the pressure cycle, even after the phase transformation. This effect has also been discussed in the section dealing with the in-situ Brillouin measurements and is in agreement with the literature as well as the results obtained on porous rutile $\mathrm{TiO}_{2}{ }^{24}$. Since the peak position depends on the sound velocity, the observed shifts may be due to phase transitions that affect the mechanical properties of the materials, especially those of the $\alpha-\mathrm{PbO}_{2}$ phase.
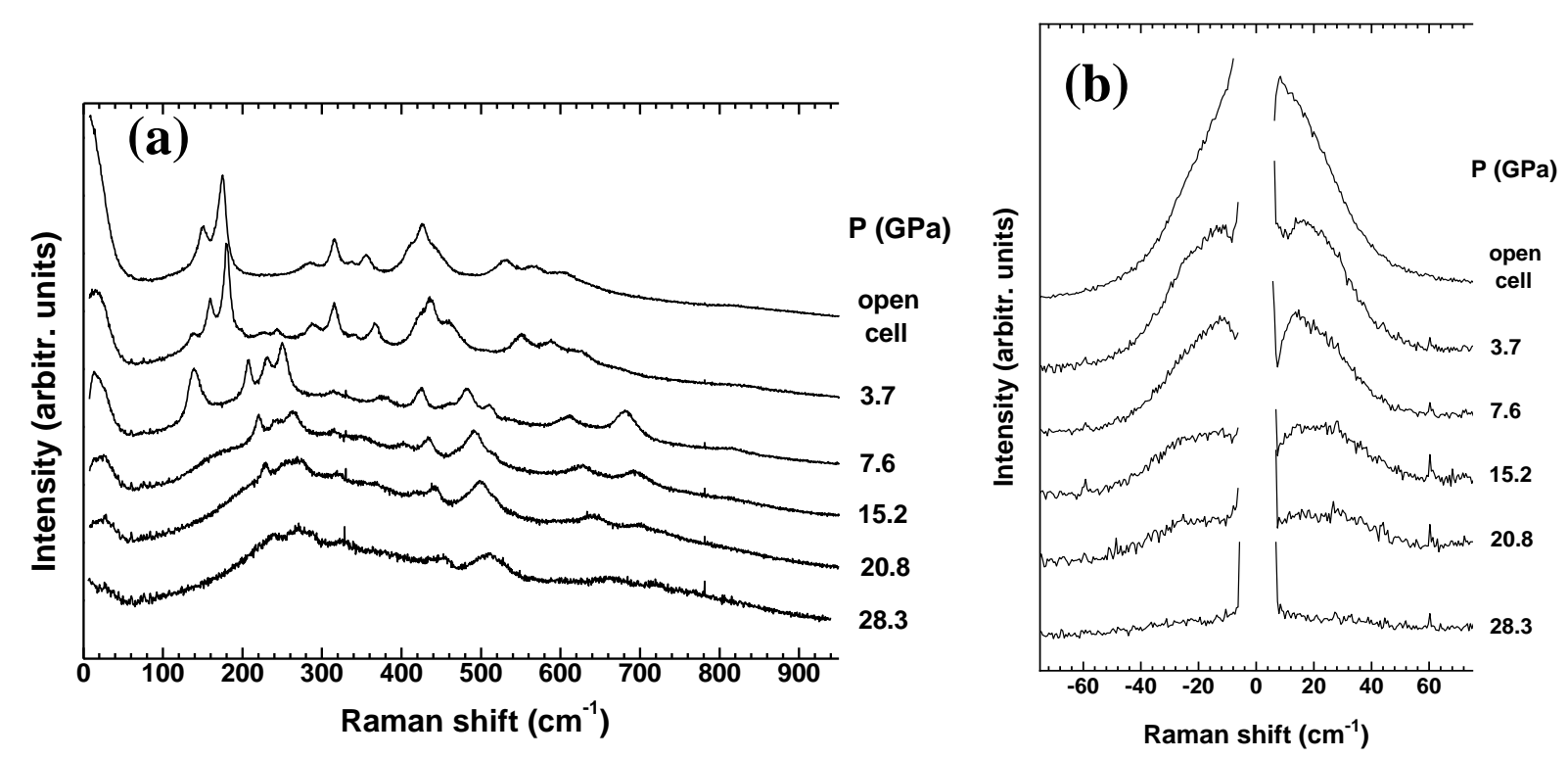

Figure 11 - Raman spectra of mesoporous $\mathrm{TiO}_{2}$ with decreasing pressure for (a) the extended $0-950 \mathrm{~cm}^{-1}$ range (b) the low-frequency range $-70 ;+70 \mathrm{~cm}^{-1}$

Phase transitions during compression and decompression are very similar to what happens in nanoparticles. First, as in the case of pressure-induced phase transitions in nanoparticles, the intermediate $\alpha-\mathrm{PbO}_{2}$ phase is not observed on the compression path, whereas bulk anatase can transform to this phase prior to the baddeleyite structure. Secondly, the shift to a higher pressure of the anatase-to-baddeleyite transition for mesoporous anatase is similar to the case of 7-nm diameter particles, as well as the sequence of phase transitions on the decompression path. In the case of nanoparticles, the interface increases the total energy of the system and the pressurization of the nanoparticles leads to the observation of several metastable states as previously reported ${ }^{22}$. Porosification also creates these interfaces that affect phase transformations. Consequently, our experimental observations can be described using a classical thermodynamics approach that includes additional interface energy ${ }^{43}$. In the case of 
mesoporous samples, by analogy with the nanoparticles, the Gibbs energy is upshifted because of the contribution of this interface energy as was discussed for porous $\mathrm{Ge}^{7}$.

\section{Conclusions}

Anatase $\mathrm{TiO}_{2}$ mesoporous material was studied under high-pressure. First, an extensive study of the mesoporous texture, especially, the porosity was carried out using different techniques, TEM, Terahertz, Brillouin and Raman spectroscopies. They indicate that the pores have an average size of $5 \mathrm{~nm}$. The porosity at ambient conditions is about $60 \%$.

Upon compression, Brillouin spectroscopy indicates that porosity changes rapidly with increasing pressure and shows a high degree of reversibility during the pressure cycle, mainly in absence of phase transitions (when pressurized up to $12 \mathrm{GPa}$ and decompressed). A phase transition is observed to a disordered baddeleyite structure. The transition starts at $\sim 15 \mathrm{GPa}$ and is completed at $\sim 25 \mathrm{GPa}$. The high-pressure evolution of the mesoporous $\mathrm{TiO}_{2}$ is very similar to the nanoparticles of $\mathrm{TiO}_{2}$ with size of $7 \mathrm{~nm}$. This similarity indicates that the interface energy controlling the phase stability has the same magnitude in both nanostructures. Therefore, mesoporous samples are a good alternative to nanoparticles in applications requiring a large area.

\section{Supporting Information.}

Schematic view of the TeraHertz-Time Domain Spectroscopy set-up; Comparison of Raman spectra of mesoporous and nanoparticles of anatase $\mathrm{TiO}_{2}$; Calculation of the quadrupolar-like vibration frequency for various shapes; Comparison of the pressure-induced variations of the Raman-active modes for nanoparticles, mesoporous $\mathrm{TiO}_{2}$ and ab initio simulations.

\section{Acknowledgment}

Authors would like to warmly thank Fernand Chassagneux for the TEM images and for fruitful discussions. 


\section{References}

1 Ye, Y.; Jo, C.; Jeong, I.; Lee, J. Functional Mesoporous Materials for Energy Applications: Solar Cells, Fuel Cells, and Batteries. Nanoscale 2013, 5, 4584.

2 Wang, L.; Ding, W.; Sun, Y. The Preparation and Application of Mesoporous Materials for Energy Storage. Mat. Res. Bull. 2016, 83, 230.

3 Tolbert, S.H.; Alivisatos, A.P. High-Pressure Structural Transformations in Semiconductor Nanocrystals. Annu. Rev. Phys. Chem. 1995, 46, 595.

4 Machon, D.; Pischedda, V.; Le Floch, S.; San-Miguel, A. Perspective: High pressure Transformations in Nanomaterials and Opportunities in Material Design. J. App. Phys. 2018, 124, 160902.

5 Machon, D.; Meersman, F.; Wilding, M.C.; Wilson, M.; McMillan, P.F. Pressure-Induced Amorphization and Polyamorphism: Inorganic and Biochemical Systems. Prog. Mat. Sci. 2014, $61,216$.

6 Deb, S.K.; Wilding, M.; Somayazulu, M.; McMillan, P.F. Pressure-Induced Amorphization and an Amorphous-Amorphous Transition in Densified Porous Silicon. Nature 2001, 414, 528.

7 Boucherif, A.; Radescu, S.; Arès, R.; Mujicà, A.; Mélinon, P.; Machon, D. Metastable States in Pressurized Bulk and Mesoporous Germanium. J. Phys. Chem. C 2018, 122, 10929.

8 Chen, X.; Mao, S.S. Titanium Dioxide Nanomaterials: Synthesis, Properties, Modifications, and Applications. Chem. Rev. 2007, 107, 2891.

9 Machon, D.; Daniel, M.; Bouvier, P.; Daniele, S.; Le Floch, S.; Mélinon, P.; Pischedda, V.

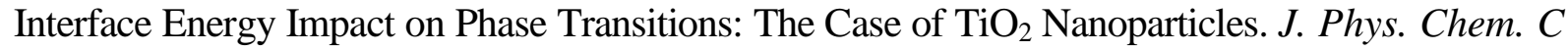
2011, 115, 22286.

10 Li, Q.; Cheng, B.; Yang, X.; Liu, R.; Liu, B.; Liu, J.; Chen, Z.; Zou, B.; Cui, T.; Liu, B. Morphology-Tuned Phase Transitions of Anatase $\mathrm{TiO}_{2}$ Nanowires under High Pressure. J. Phys. Chem. C 2013, 117, 8516.

11 Li, Q.; Liu, B.; Wang, L.; Li, D.; Liu, R.; Zou, B.; Cui, T.; Zou, G.; Meng, Y.; Mao, H.K. et al. Pressure-Induced Amorphization and Polyamorphism in One-Dimensional Single-Crystal $\mathrm{TiO}_{2}$ Nanomaterials. J. Phys. Chem. Lett. 2010, 1, 309.

12 Machon, D.; Le Bail, N.; Hermet, P.; Cornier, T.; Daniele, S.; Vignoli, S. Pressure-Induced Phase Transitions in $\mathrm{TiO}_{2}$ Rutile Nanorods. J. Phys. Chem. C 2019, 123, 1948.

13 Nicol, M.; Fong, M.Y. Raman Spectrum and Polymorphism of Titanium Dioxide at High Pressures. J. Chem. Phys. 1971, 54, 3167. 
14 Mammone, J.F.; Sharma, S.K.; Nicol, M. Raman Spectra of Titanium Dioxide. Solid State Com. 1980, 34, 799.

15 Arashi, H. Raman Spectroscopic Study of the Pressure-Induced Phase Transition in $\mathrm{TiO}_{2}$. J. Phys. Chem. Solids 1992, 53, 355.

16 Sato, H.; Endo, S.; Sugiyama, M.; Kikegawa, T.; Shimomura, O.; Kusaba, K. BaddeleyiteType High-Pressure Phase of $\mathrm{TiO}_{2}$. Science 1991, 251, 786.

17 Gerward, L.; Olsen, J.S. Post-Rutile High-Pressure Phases in $\mathrm{TiO}_{2}$. J. Appl. Cryst. 1997, 30, 259.

18 Navrotsky, A. Energetics of Nanoparticle Oxides: Interplay between Surface Energy and Polymorphism. Geochem. Trans. 2003, 4, 34.

19 Popescu, C.; Sans, J.A.; Errandonea, D.; Segura, A.; Villanueva, R.; Sapiñ, F. Compressibility and Structural Stability of Nanocrystalline $\mathrm{TiO}_{2}$ Anatase Synthesized from FreezeDried Precursors. Inorg. Chem. 2014, 53, 11598.

20 Pischedda, V.; Hearne, G.R.; Dawe, A.M.; Lowther, J.E. Ultrastability and Enhanced Stiffness of $\sim 6 \mathrm{~nm} \mathrm{TiO}{ }_{2}$ Nanoanatase and Eventual Pressure-Induced Disorder on the Nanometer Scale. Phys. Rev. Lett. 2006, 96, 035509.

21 Swamy, V.; Kuznetsov, A.; Dubrovinsky, L.S.; McMillan, P.F.; Prakapenka, V.B.; Shen, G.; Muddle, B.C. Size-Dependent Pressure-Induced Amorphization in Nanoscale $\mathrm{TiO}_{2}$. Phys. Rev. Lett. 2006, 96, 135702.

22 Machon, D.; Daniel, M.; Pischedda, V.; Daniele, S.; Bouvier, P.; LeFloch, S. PressureInduced Polyamorphism in $\mathrm{TiO}_{2}$ Nanoparticles. Phys. Rev. B 2010, 82, 140102.

23 Machon, D.; Mélinon, P. Size-Dependent Pressure-Induced Amorphization: a Thermodynamic Panorama. Phys. Chem. Chem. Phys. 2015, 17, 903.

24 Li, Q.; Liu, R.; Liu, B.; Wang, L.; Wang, K.; Li, D.; Zou, B.; Cui, T.; Liu, J.; Chen, Z.; et al. Stability and Phase Transition of Nanoporous Rutile $\mathrm{TiO}_{2}$ under High Pressure. RSC Advances 2012, 2, 9052 .

25 Li, Q.; Liu, R.; Cheng, B.; Wang, L.; Yao, M.; Li, D.; Zou, B.; Cui, T.; Liu, B. High Pressure Behaviors of Nanoporous Anatase $\mathrm{TiO}_{2}$. Mat. Res. Bull. 2012, 47, 1396.

26 Brinker, C.J.; Lu, Y.; Sellinger, A.; Fan, H. Evaporation- Induced Self- Assembly: Nanostructures Made Easy. Adv. Mater. 1999, 11, 579.

27 Liu, K.; Fu, H.; Shi, K.; Xiao, F.; Jing, L.; Xin, B. Preparation of Large-Pore Mesoporous Nanocrystalline $\mathrm{TiO}_{2}$ Thin Films with Tailored Pore Diameters. J. Phys. Chem. B 2005, 109, 18719. 
28 Soler-Illia, G.J. de A.A.; Louis, A.; Sanchez, C. Synthesis and Characterization of Mesostructured Titania-Based Materials through Evaporation-Induced Self-Assembly. Chem. Mater. 2002, 14, 750 .

29 Bois, L.; Chassagneux, F.; Battie, Y.; Bessueille, F.; Mollet, L.; Parola, S.; Destouches, N.; Toulhoat, N.; Moncoffre, N. Chemical Growth and Photochromism of Silver Nanoparticles into a Mesoporous Titania Template. Langmuir 2010, 26, 1199.

30 Couzon, N.; Maillard, M.; Bois, L.; Chassagneux, F.; Brioude, A. Electrochemical Observation of the Plasmonic Effect in Photochromic Ag Nanoparticle Filled Mesoporous $\mathrm{TiO}_{2}$ Films. J. Phys. Chem. C 2017, 121, 22147.

31 See Supporting Information.

32 Otto, J. W.; Vassiliou, J. K.; Frommeyer, G. Non Hydrostatic Compression of Elastically Anisotropic Polycrystals. I. Hydrostatic Limits of 4:1 Methanol-Ethanol and Paraffin Oil. Phys. Rev. B: Condens. Matter Mater. Phys. 1998, 57, 3253. (35)

33 Thommes, M.; Kaneko, K.; Neimark, A.V.; Olivier, J.P.; Rodriguez-Reinoso, F.; Rouquerol, J.; Sing, K.S.W. Physisorption of Gases, with Special Reference to the Evaluation of Surface Area and Pore Size Distribution (IUPAC Technical Report). Pure Appl. Chem. 2015, 87, 1051 .

34 Siefke, T.; Kroker, S.; Pfeiffer, K.; Puffky, O.; Dietrich, K.; Franta, D.; Ohlídal, I.; Szeghalmi, A.; Kley, E.-B.; Tünnermann, A. Materials Pushing the Application Limits of Wire Grid Polarizers Further into the Deep Ultraviolet Spectral Range. Adv. Opt. Mater. 2016, 4, 1780.

35 Markel. V.A. Introduction to the Maxwell Garnett Approximation: Tutorial. J. Opt. Soc. Am. A 2016, 33, 1244.

36 Fan, H.J.; Kuok, M.H.; Ng, S.C.; Boukherroub, R.; Baribeau, J.M.; Fraser, J.W.; Lockwood, D.J. Brillouin Spectroscopy of Acoustic Modes in Porous Silicon Films. Phys. Rev. B 2002, 65, 165330 .

37 Ohsaka, T.; Izumi, F.; Fujiki, Y. Raman Spectrum of Anatase, $\mathrm{TiO}_{2}$. J. Raman Spectrosc. 1978, 7, 321 .

38 Pighini, C.; Aymes, D.; Millot, N.; Saviot, L. Low-Frequency Raman Characterization of Size-Controlled Anatase $\mathrm{TiO}_{2}$ Nanopowders Prepared by Continuous Hydrothermal Syntheses. $J$. Nanopart. Res. 2006, 9, 309.

39 Saviot, L. Vibrations of Single-Crystal Gold Nanorods and Nanowires. Phys Rev B 2018, 97, 155420. 
40 Girard, A.; Gehan, H.; Crut, A.; Mermet, A.; Saviot, L.; Margueritat, J. Mechanical Coupling in Gold Nanoparticles Supermolecules Revealed by Plasmon-Enhanced Ultralow Frequency Raman Spectroscopy. Nano Lett. 2016, 16, 3843.

41 Nowak, E.; Combes, G.; Hugh Stitt, E.; Pacek, A.W. A Comparison of Contact Angle Measurement Techniques Applied to Highly Porous Catalyst Supports. Powder Technology 2013, 233, 52 .

42 Saviot, L.; Machon, D.; Debbichi, L.; Girard, A.; Margueritat, J.; Krüger, P.; Marco de Lucas, M.C.; Mermet, A. Optical and Acoustic Vibrations Confined in Anatase $\mathrm{TiO}_{2}$ Nanoparticles under High-Pressure. J. Phys. Chem. C 2014, 118, 10495.

43 Piot, L.; Le Floch, S.; Cornier, T.; Daniele, S.; Machon, D. Amorphization in Nanoparticles. J. Phys. Chem. C 2013, 117, 11133.

\section{TOC graphic}

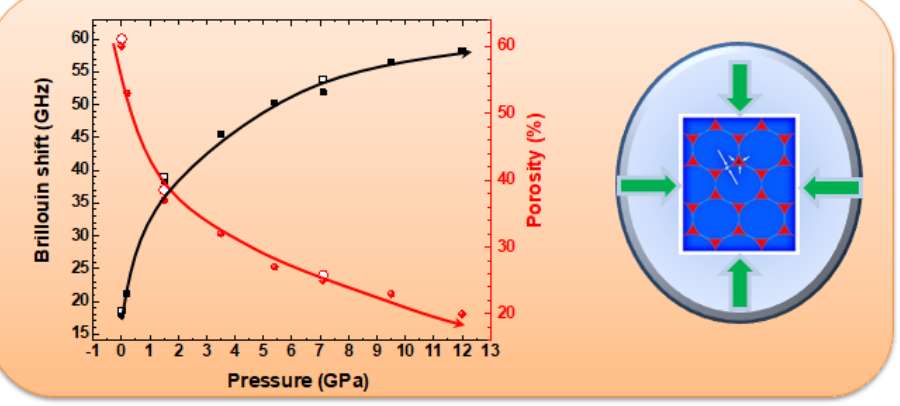

